\title{
Engineering the binding kinetics of synthetic polymer nanoparticles for siRNA delivery
}

Hiroyuki Koide, $\dagger$ Tatsuya Fukuta,$\dagger$ Anna Okishim, $\dagger$ Saki Ariizumi, $\dagger$ Chiaki Kiyokawa, $\dagger$

Hiroki Tsuchida, $\dagger$ Masahiko Nakamoto $\neq$ Keiichi Yoshimatsu, $§$ Hidenori Ando, $\dagger$ Takehisa Dewa, // Tomohiro Asai, $\dagger$ Naoto Oku, $\dagger$ Yu Hoshino, $*+$ and Kenneth J. Shea* $\S$

$\dagger$ Department of Medical Biochemistry, School of Pharmaceutical Sciences, University of

Shizuoka, 52-1 Yada, Suruga-ku, Shizuoka, Shizuoka 422-8526, Japan; $\ddagger$ Department of

Chemical Engineering, Kyushu University, 744 Motooka, Fukuoka 819-0395, Japan; §

Department of Chemistry, University of California Irvine, Irvine, CA 92697 USA; II

Department of Life Science and Applied Chemistry, Graduate School of Engineering,

Nagoya Institute of Technology, Gokiso-cho, Showa-ku, Nagoya, Aichi 466-8555, Japan

\section{Supporting Information}




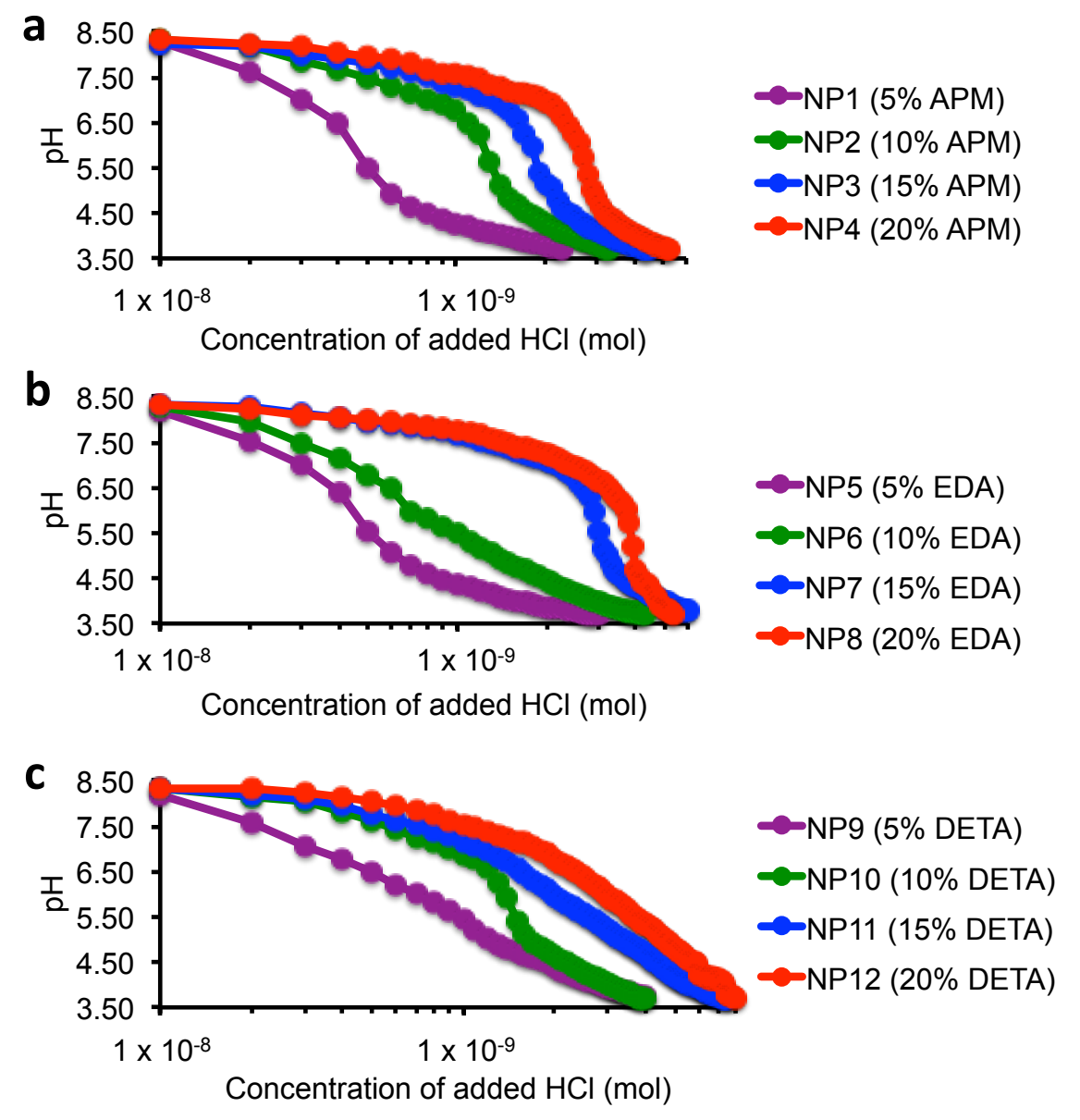

Figure S1. Titration assay of amine NPs. $0.01 \mathrm{~N} \mathrm{HCl}$ was added into $1 \mathrm{mg} / \mathrm{ml}$ of (a) APM,

(b) EDA or (c) DETA NPs. Then, pH changes were measured. 
a

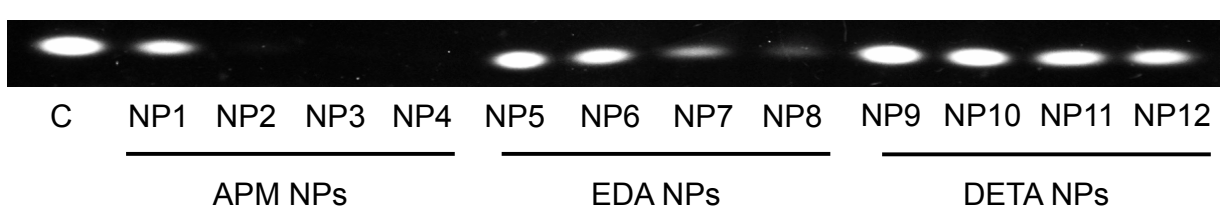

b

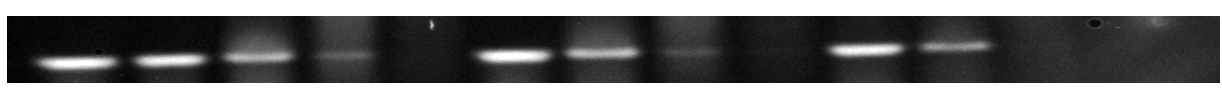

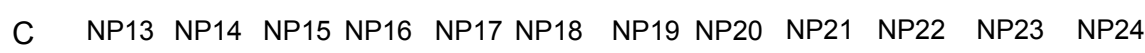
$0 \%$ TBAm 10\% TBAm $20 \%$ TBAm

C

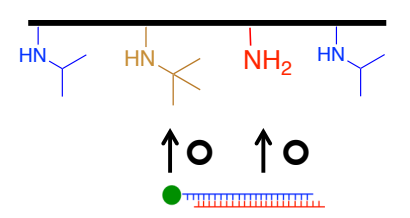

d
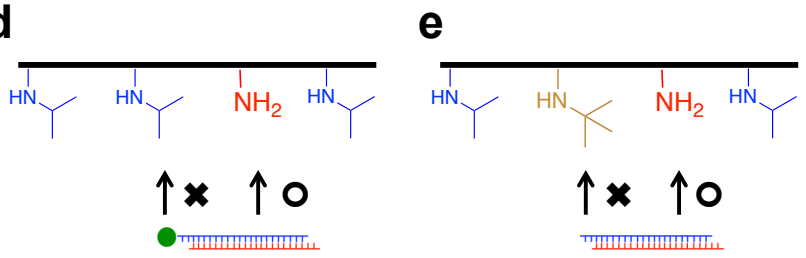

Figure S2. Importance of Hydrophobic interaction between NPs and siRNA. (a) Interaction between cholesterol free siRNA and amine NPs. (b), Interaction between siRNA-C and several percentage of TBAm NPs (0-20\%). C: Naked cholesterol free siRNA (a) or naked siRNA-C (b) (c-e), Schematic representation of NP-siRNA-C interactions. Contributions of hydrophobic and electrostatic interactions between (c) NP and siRNA-C, (d) TBAm decreased NPs and siRNA-C or (e) NPs and non-siRNA-C. 

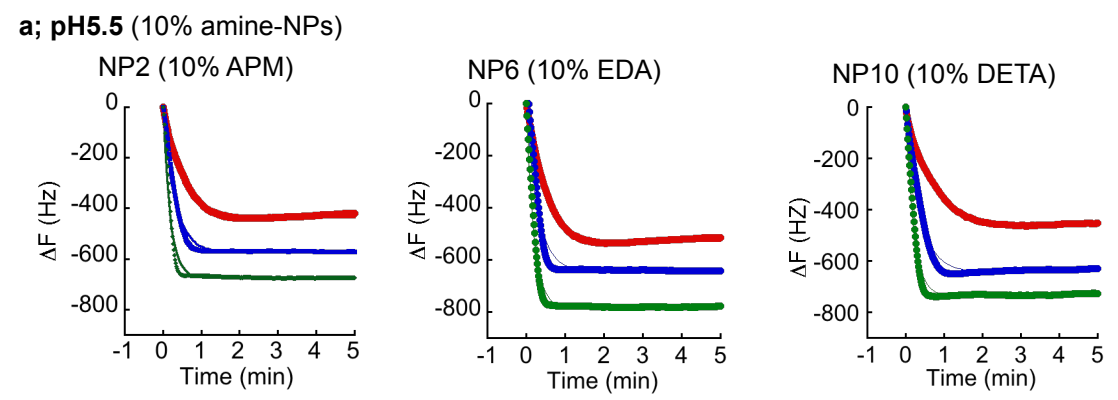

b; pH5.5 (20\% amine-NPs)
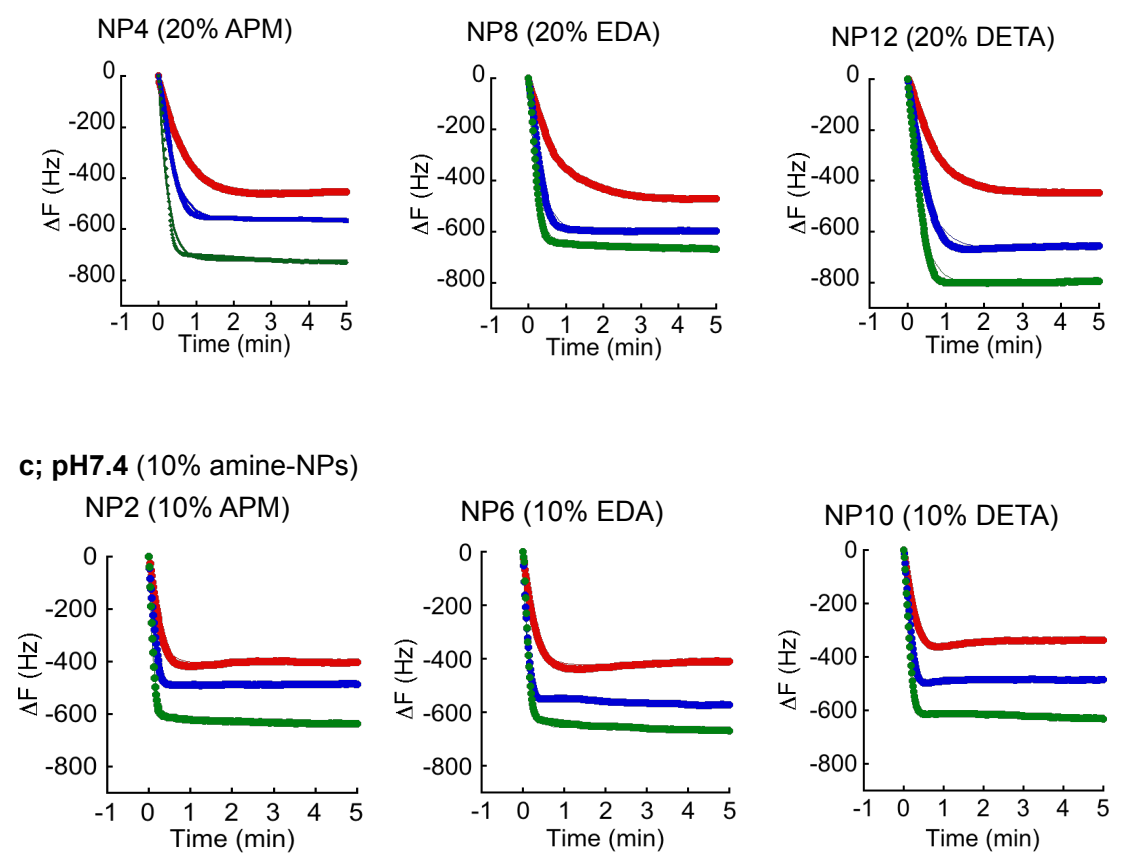

d; pH7.4 (20\% amine-NPs)
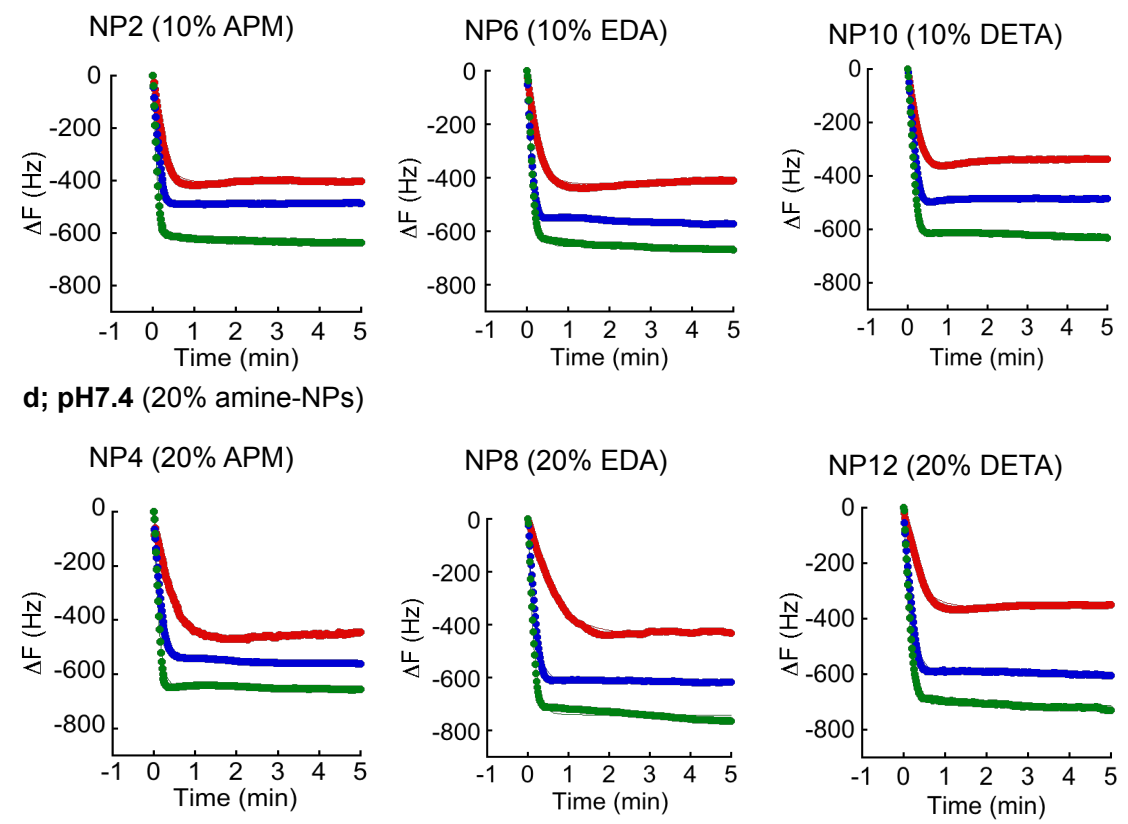

Figure S3. Time courses of frequency changes of NP2 (10\% APM), 4 (20\% APM), 6 (10\% EDA), 8 (20\% EDA), 10 (10\% DETA) or 12 (20\% DETA)-immobilized QCM after addition of several concentrations of siRNA-C at pH5.5 or 7.4. Data indicate red; $30 \mathrm{nM}$, blue; 100 $\mathrm{nM}$, green; $300 \mathrm{nM}$. Results of triplicated experiments (thick lines) and fit curves (thin lines) are overwritten. 

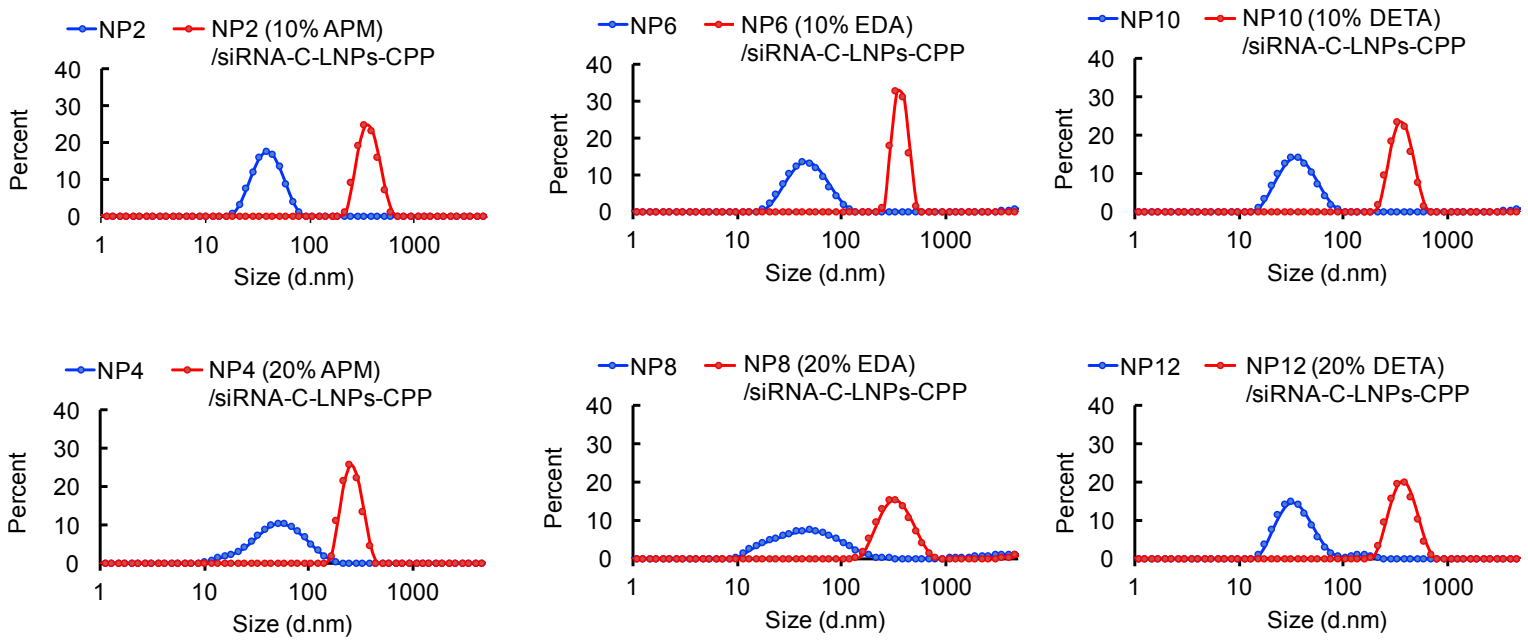

Figure S4. Size distributions of NPs and NP/siRNA-C-LNP-CPP. 


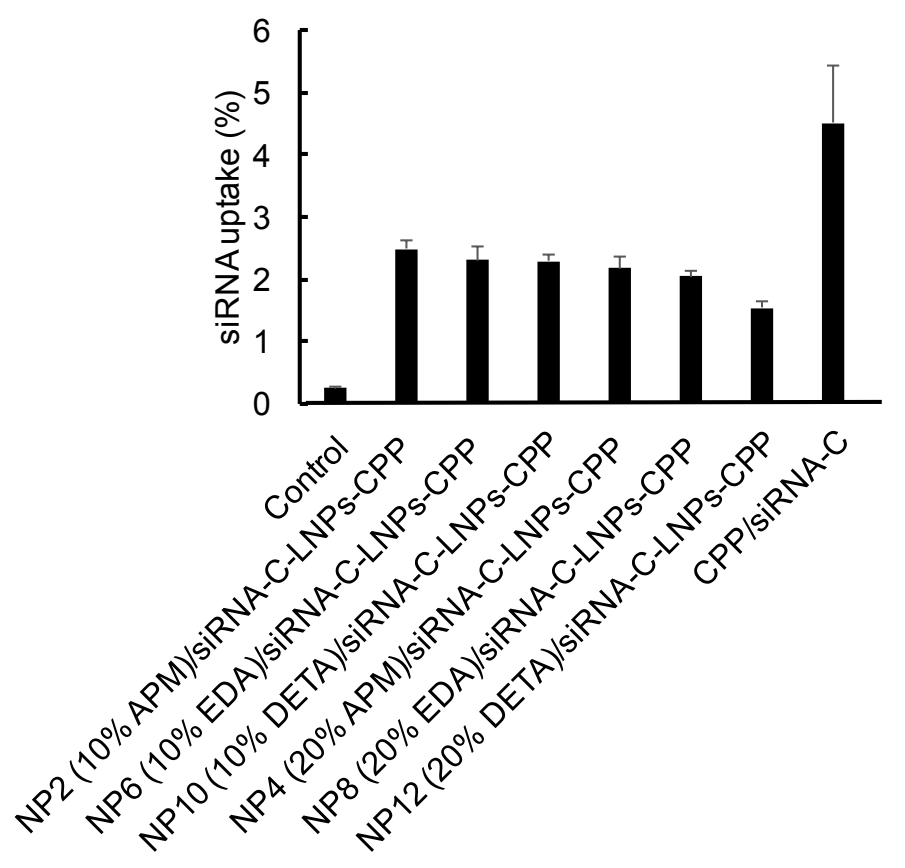

Figure S5. siRNA uptake using NP/siRNA-C-LNP-CPP.

Twenty-four hours after the addition of NP/siRNA-C-LNP-CPP to the cells, fluorescence intensity of FITC-siRNA-C in the cells was measured. CPP and siRNA complex was measured as a positive control. 
a

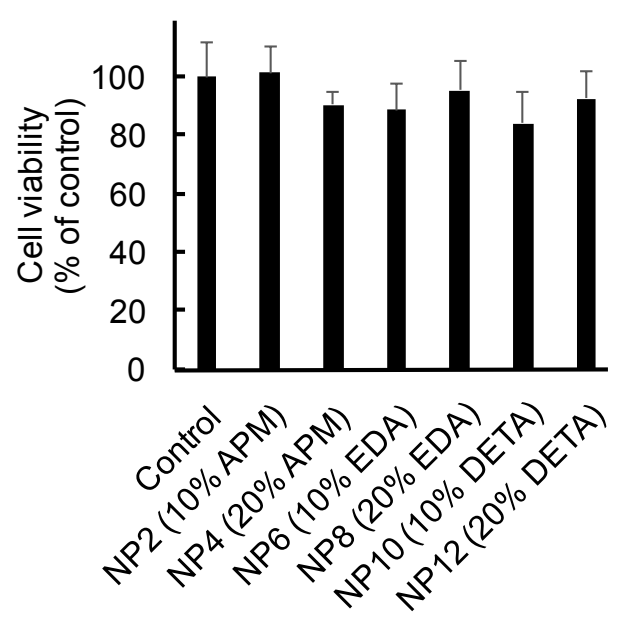

b
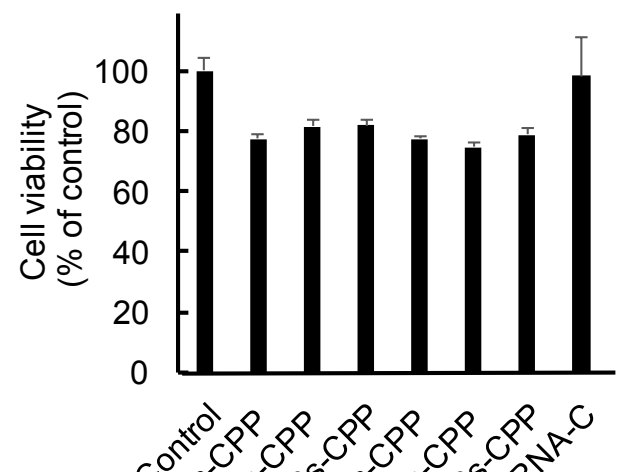

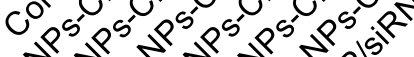

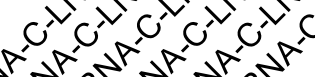

. ल5.

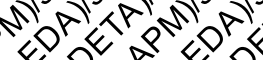

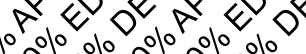

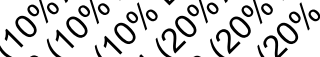
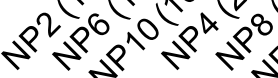

Figure S6. Cytotoxicity assay of NPs or NP/siRNA-C-LNP-CPP.

B16F10-Luc2 cells were transfected with (a) NPs or (b) NPs/siRNA-C-LNP-CPP. At $48 \mathrm{~h}$ after the addition, protein content of the living cells was measured by use of the BCA protein assay. 
(a)

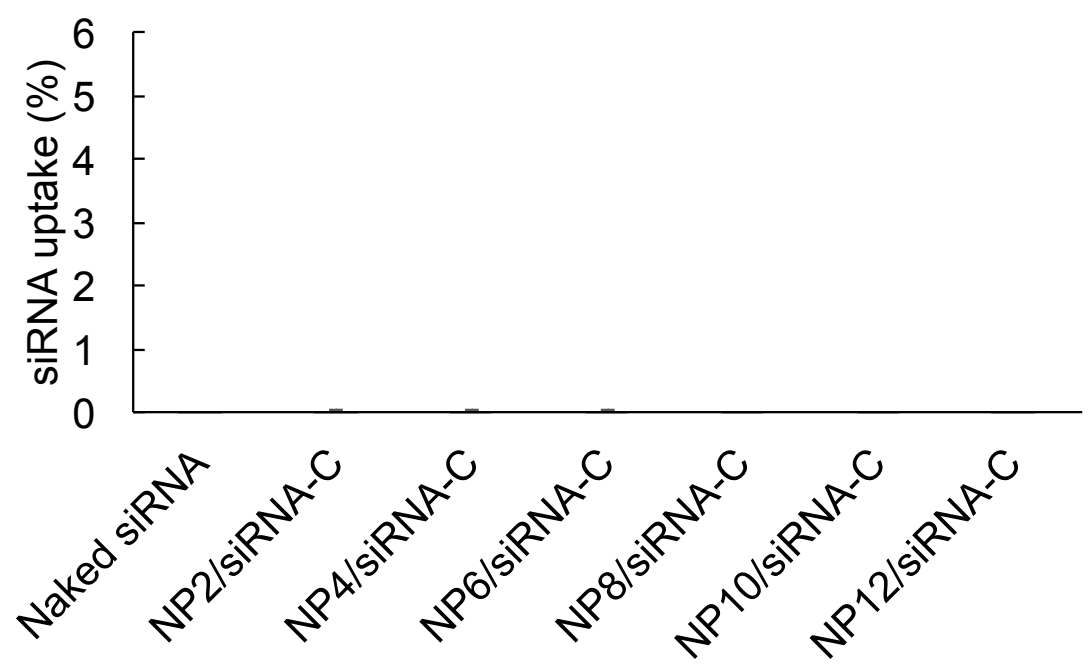

(b)

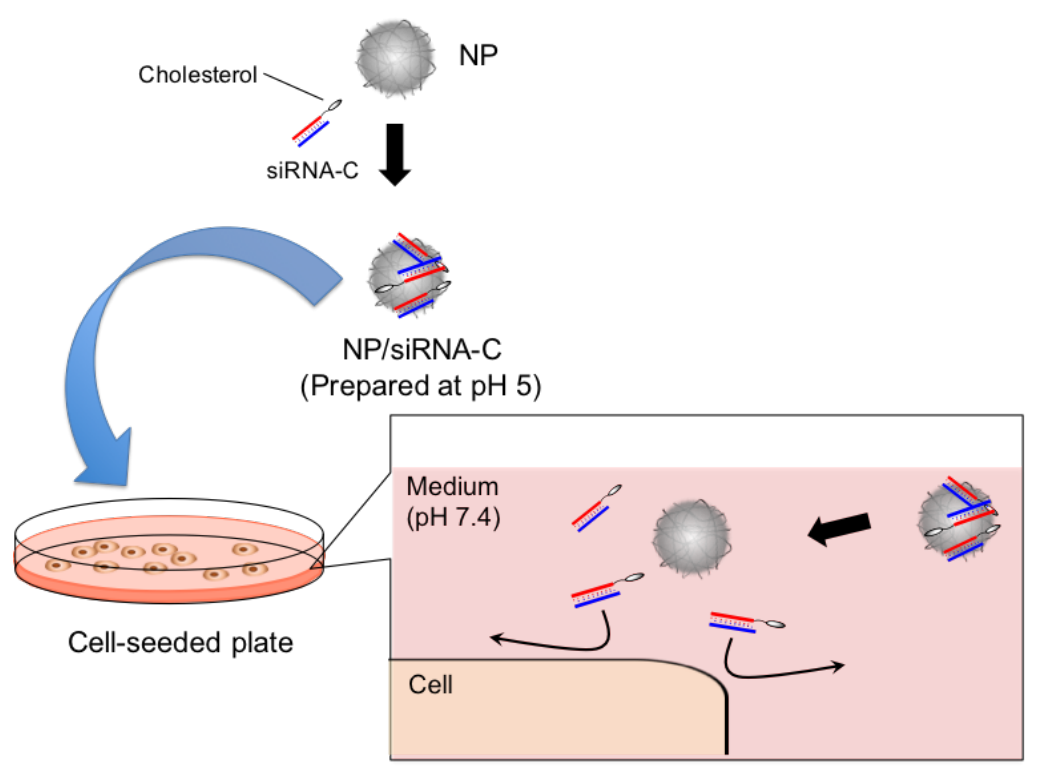

Figure S7. (a) siRNA uptake using NP/siRNA. Twenty-four hours after the addition of NP/siRNA to the cells, fluorescence intensity of FITC-siRNA-C in the cells was measured. (b) Naked NP can not deliver siRNA into the cell because naked NPs can not stably hold siRNA in the culture medium ( $\mathrm{pH}$ 7.4) but release it into the medium. " 


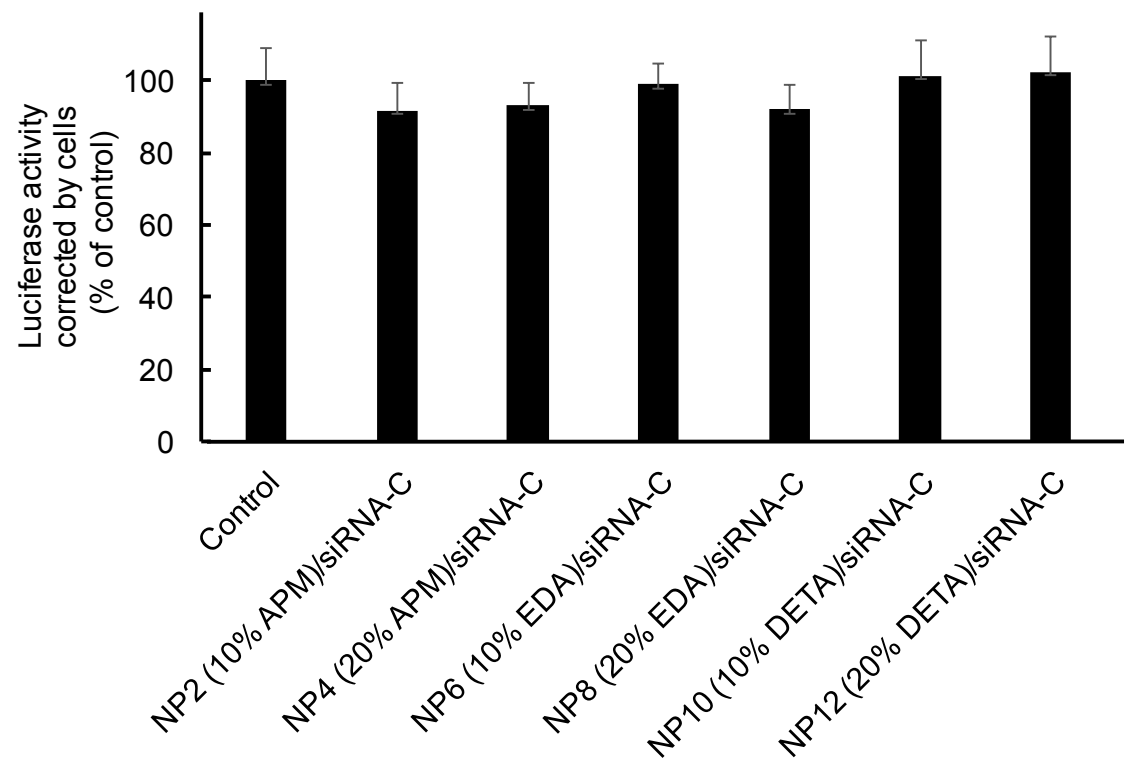

Figure S8. Knockdown effect of NP/siRNA-C complex.

B16F10-Luc2 cells were transfected with NP/siRNA-C. At $48 \mathrm{~h}$ after the addition, luciferase activities were measured and normalized by the total protein content. 
Table S1. Monomer compositions, sizes, $\zeta$-potentials and electrostatic mobility of NPs.

\begin{tabular}{|c|c|c|c|c|c|c|c|c|}
\hline & NIPAm & DETA & TBAm & BIS & Size $(n m)$ & PDI & $\zeta$-potential $(\mathrm{mV})$ & Mobility $(\mu \mathrm{mcm} / \mathrm{Vs})$ \\
\hline NP13 & 93 & 5 & 0 & 2 & 58.5 & 0.19 & 14.0 & 1.1 \\
\hline NP14 & 88 & 10 & 0 & 2 & 99.3 & 0.28 & 25.3 & 2.0 \\
\hline NP15 & 83 & 15 & 0 & 2 & 141.8 & 0.31 & 27.3 & 2.1 \\
\hline \multirow[t]{2}{*}{ NP16 } & 78 & 20 & 0 & 2 & 196.1 & 0.30 & 33.7 & 2.6 \\
\hline & NIPAm & DETA & TBAm & BIS & Size $(n m)$ & PDI & $\zeta$-potential $(\mathrm{mV})$ & Mobility $(\mu \mathrm{mcm} / \mathrm{Vs})$ \\
\hline NP17 & 83 & 5 & 10 & 2 & 43.3 & 0.17 & 19.2 & 1.5 \\
\hline NP18 & 78 & 10 & 10 & 2 & 42.9 & 0.19 & 25.0 & 2.0 \\
\hline NP19 & 73 & 15 & 10 & 2 & 50.8 & 0.29 & 28.8 & 2.3 \\
\hline \multirow[t]{2}{*}{ NP20 } & 58 & 20 & 10 & 2 & 140.3 & 0.32 & 27.4 & 2.1 \\
\hline & NIPAm & DETA & TBAm & BIS & Size $(n m)$ & PDI & $\zeta$-potential $(\mathrm{mV})$ & Mobility $(\mu \mathrm{mcm} / \mathrm{Vs})$ \\
\hline NP21 & 73 & 5 & 20 & 2 & 44.6 & 0.13 & 32.4 & 2.5 \\
\hline NP22 & 68 & 10 & 20 & 2 & 36.6 & 0.17 & 34.2 & 2.7 \\
\hline NP23 & 53 & 15 & 20 & 2 & 36.0 & 0.27 & 36.6 & 2.9 \\
\hline NP24 & 58 & 20 & 20 & 2 & 45.0 & 0.40 & 23.5 & 1.8 \\
\hline
\end{tabular}

